Pacific Journal of Mathematic 


\section{A CLASS OF MEASURES ON THE BOHR GROUP}

\section{MAHENDRA NADKARNI}

Let $R_{d}$ denote the real line with the discrete topology. Let $B=\hat{R}_{d}$ be its dual. $R$, the real line is continuously isomorphic to a dense subgroup of $B$. Let $\mu$ be a finite positive measure defined for Borel subsets of $B$. Let $\chi_{t}$ denote the character on $B$ corresponding to the real number $t$. We shall denote by $H_{S}$ the subspace of $L_{2}(B, \mu)$ spanned by $\left\{\chi_{t}: t \leqq S\right\}$. Assume that $\bigcap_{-\infty<S<\infty} H_{S}=\{0\}$. In this case the subspaces $H_{S}$ are strictly increasing in the sense that $H_{S} \varsubsetneqq H_{S^{\prime}}$ whenever $S<S^{\prime}$. The increasing subspaces generate a spectral measure $E$ defined for intervals $a<x \leqq b$ by $E(a, b]=$ orthogonal projection on $H_{b} \ominus H_{a}$. We shall say that $E$ has multiplicity 1 if there exists an element $w \in L_{2}(B, \mu)$ such that $\{E(\sigma) w: \sigma \in \mathscr{B}\}$ spans $L_{2}(B, \mu)$. Here $\mathscr{B}$ denotes the class of Borel subsets of $R$.

Theorem 1. Assume that

(i) $\bigcap_{S} H_{S}=\{0\}$.

(ii) $E$ has multiplicity 1.

Then $\mu$ sits on a coset of $R$ in $B$.

The present work was suggested and is strongly influenced by papers of Helson and Lowdenslager [6, 7] and Helson [4, 5]. Other papers that were useful are also listed in the references.

2. In this section we shall state some general results about spectral measures pertinent to us and prove some results that shall be of use. Proof of Theorem 1 will be given in $\S 3$.

Let $H$ be a (complex) Hilbert space. Let $E$ be a spectral measure defined on Borel subsets $\mathscr{B}$ of the real line. Values of $E$ are orthogonal projections on subspaces of $H$. Suppose that $E$ has multiplicity 1, i.e., there exists a vector $z \in H$ such that $\{E(\sigma) z: \sigma \in \mathscr{B}\}$ spans $H$. This implies that $H$ is separable. Such a $z$ is called cyclic vector for $E$. Write $m(\sigma)=\|E(\sigma)\|^{2}, \sigma \in \mathscr{B}$. Then $m$ is a finite positive measure on $\mathscr{B}$. If we write $z(\sigma)=E(\sigma) z$, then $z(\sigma)$ is a vector valued measure taking values in $H$. It can be shown that under the assumption of multiplicity 1 , every element $h \in H$ has an integral representation of the type $h=\int_{-\infty}^{\infty} \varphi_{h}(\lambda) d z(\lambda)$, with

$$
\int_{-\infty}^{\infty}\left|\varphi_{h}(\lambda)\right|^{2} d m(\lambda)<\infty
$$

[§1, p. 264]. Further $E(\sigma) h=\int_{\sigma} \varphi_{h}(\lambda) d z(\lambda)$. Let $h_{1}, h_{2}, h_{3}, \cdots$ be a 
complete orthonormal set in $H$. Let measures $m_{i}, i=1,2,3, \cdots$ be defined by $m_{i}(\sigma)=\left\|E(\sigma) h_{i}\right\|^{2}$. Let $\nu$ denote the measure $\nu(\sigma)=$ $\sum_{i=1}^{\infty}\left(1 / 2^{n}\right) m_{i}(\sigma)$. The following lemma is known and easy to prove.

Lemma 1. If $E$ has multiplicity 1 and $z$ its cyclic vector, then measure $m$ and $\nu$ are mutually absolutely continuous. Here $m$ is the measure defined by $m(\sigma)=\|E(\sigma) z\|^{2}$.

The next lemma is known and its proof can be found in ([9], p. 318). For any measure $\mu_{0}$ we shall write $\mu_{t}$ for the measure $\ell_{t}(\sigma)=$ $\mu(\sigma+t), \sigma \in \mathscr{B}, t \in R$.

Lemma 2. $\mu_{0}$ is a finite positive measure defined on Borel subsets of $R$. Assume that $\mu_{0}$ and $\mu_{t}$ are mutually absolutely continuous. Then $\mu_{0}$ and Lebesgue measure are mutually absolutely continuous.

A function called cocycle presents itself in the proof of Lemma 5. It is necessary to show that, in our context, such function has a special form called coboundary.

DeFinition 1. A function $A(t, \lambda)$ on $R \times R$ is called a cocycle if (i ) $|A(t, \lambda)|=1$ for all $t, \lambda$.

(ii) $A(t, \lambda)$ is Borel measurable in $\lambda$ for every fixed $t$.

(iii) $A(t+u, \lambda) A(t, \lambda) A(u, \lambda+t)$ for almost every $\lambda \in R$ (with respect to the Lebesgue measure).

The set of Lebesgue measure zero where (iii) does not hold may depend on $(t, u)$.

Definition 2. A cocycle $A(t, \lambda)$ is called a coboundary if it is of the type $B(\lambda+t) B^{-1}(\lambda)$ for some function $B$ on $R$ of absolute value 1 .

We shall prove the following

Lemma 3. Every cocycle is a coboundary.

Few remarks should be made before we prove this lemma. The proof of Lemma 3 is trivial if condition (iii) of cocycle held everywhere instead of almost everywhere. For in that case we need only put $\lambda=0$ in (iii) and observe that

$$
A(u, t)=A(t+u, 0) A^{-1}(t, 0) .
$$

Cocycles and coboundaries occur very crucially in the works of Henry Helson and David Lowdensager although domain of definitions of these functions changes according to context in their work. In his 
book on invariant subspace [4], Helson proved Lemma 3 under an additional hypothesis which is equivalent to requiring that $\Lambda(t, \lambda)$ be jointly measurable in $(t, \lambda)$. There are cases however where one has to deal with cocycles $A(t, \lambda)$ which are measurable in $\lambda$ for every fixed $t$. The present paper is one such case.

Finally we remark that the idea of our proof is already contained in the papers of Mackey [9] and Helson [4, 5]. We state here, without proof, a theorem of Mackey [9, p. 317] which we shall need in the proof of Lemma 3.

THEOREM 2. Let $M_{1}$ and $M_{2}$ be sigma finite measure spaces with measures $\mu_{1}$ and $\mu_{2}$. Suppose that there is a countably generated Borel field $\mathscr{C}$ of measurable subsets of $M_{2}$ such that every measurable subset of $M_{2}$ differs from some member of $\mathscr{C}$ by subset of a set of measure zero. Let $f$ be a complex valued function on $M_{1} \times M_{2}$ which is measurable and essentially bounded as a function on $M_{2}$ for each fixed point in $M_{1}$. Suppose that $\int_{E} f(x, y) d \mu_{2}(y)$ is measurable on $M_{1}$ for each fixed measurable subset $E^{\prime}$ of $M_{2}$ of finite measure. Then there exists a function $f^{\prime}(x, y)$ jointly measurable on $M_{1} \times M_{2}$ such that for all $x \in M_{1}$

$$
f(x, y)=f^{\prime}(x, y)
$$

for almost all $y \in M_{2}$.

Proof of Lemma 3. Consider

$$
F(u, t, \lambda)=A(t+u, \lambda) A^{-1}(t, \lambda) A^{-1}(u, \lambda) .
$$

From the cocycle relations (iii) we see that for each fixed $u, t$

(1) $F(u, t, \lambda)=A(u, \lambda+t) A^{-1}(u, \lambda) \quad$ a.e. $\lambda$.

(2) $F(u, t, \lambda)=A(t, \lambda+u) A^{-1}(t, \lambda) \quad$ a.e. $\lambda$.

(1) and (2) show that $F(u, t, \lambda)$ is measurable in $(t, \lambda)$ for each fixed $u$ and measurable in $(u, \lambda)$ for each fixed $t$. We show that $F$ can be chosen to be measurable in all three variable $(u, t, \lambda)$ and still satisfy (1) and (2). Let $\sigma$ be a measurable set in $(t, \lambda)$ of finite measure. Consider

$$
\iint_{\sigma} F(u, t, \lambda) d \lambda d t
$$

We show that this integral moves continuously in $u$.

$$
\begin{aligned}
\iint_{\sigma} \mid & F(u, t, \lambda)-F(s, t, \lambda) \mid d \lambda d t \\
= & \iint_{\sigma} \mid A(t+u, \lambda) A^{-1}(t, \lambda) A^{-1}(u, \lambda) \\
& \quad-A(t+s, \lambda) A^{-1}(t, \lambda) A^{-1}(s, \lambda) \mid d \lambda d t \\
= & \iint_{\sigma}\left|A(t+u, \lambda) A^{-1}(u, \lambda)-A(t+s, \lambda) A^{-1}(s, \lambda)\right| d \lambda d t .
\end{aligned}
$$


This identity holds since $|A(t, \lambda)|=1$. Again by cocycle identity (iii), $\left(^{*}\right)$ is equal to

$$
\iint_{\sigma}|A(t, \lambda+u)-A(t, \lambda+s)| d \lambda d t \rightarrow 0 \quad \text { as } s \rightarrow u .
$$

Thus for each $(t, \lambda)$ measurable set $\sigma$ of finite measure

$$
\iint_{\sigma} F(u, t, \lambda) d \lambda d t
$$

moves continuously in $u$. So by Theorem 2 , we can replace $F(u, t, \lambda)$ by a measurable function in all three variables.

So we assume now that $F$ is measurable in all three variables. Now

$$
\left.{ }^{* *}\right) \quad A(t+u, \lambda) A^{-1}(t, \lambda) A^{-1}(u, \lambda)=A(u, \lambda+t) A^{-1}(u, \lambda)
$$

for almost every $\lambda$ for fixed $(t, u)$. But the left hand side is measurable in $(u, t, \lambda)$ and so by Fubini theorem there exists $\lambda_{0}$ such that $\left({ }^{* *}\right)$ holds for almost every $(u, t)$. So for almost every $(u, t)$

$$
\begin{aligned}
A\left(t+u, \lambda_{0}\right) A^{-1}\left(t, \lambda_{0}\right) A^{-1}\left(u, \lambda_{0}\right) & =A\left(u, \lambda_{0}+t\right) A^{-1}\left(u, \lambda_{0}\right) \\
A\left(t+u, \lambda_{0}\right) A^{-1}\left(t, \lambda_{0}\right) & =A\left(u, \lambda_{0}+t\right) .
\end{aligned}
$$

Put $\lambda_{0}+t=s$, then

$$
A\left(s-\lambda_{0}+u, \lambda_{0}\right) A^{-1}\left(s-\lambda_{0}, \lambda_{0}\right)=A(u, s) .
$$

Write $B(x)=A\left(x-\lambda_{0}, \lambda_{0}\right)$. Obviously $A(u, s)=B(u+s) B^{-1}(s)$.

REMARK. We note that

$$
B(u+s) B^{-1}(s) B^{-1}(u)=A\left(u+s-\lambda_{0}, \lambda_{0}\right) A^{-1}\left(s-\lambda_{0}, \lambda_{0}\right) A^{-1}\left(u-\lambda_{0}, \lambda_{0}\right)
$$

is jointly measurable in $(u, s)$.

Definition. A spectral measure $E$ on $\mathscr{B}$ is called stationary if there exists a commutative group $T^{t}, t \in R$, of unitary operators such that for every Borel set $\sigma \in \mathscr{B}, T^{t} E(\sigma) T^{-t}=E(\sigma+t)$.

Lemma 4. Let $E$ be a stationary spectral of multiplicity 1 and let $z$ be a cyclic vector for $E$. Then the measure $m$ defined by $m(\sigma)=\|E(\sigma) z\|^{2}$ and Lebesgue measure are mutually absolutely continuous.

Proof. Let $h_{1}, h_{2}, h_{3}, \cdots$ be a complete orthonormal set in $H$. Since $T^{t}$ is unitary $T^{t} h_{1}, T^{t} h_{2}, \cdots$ is again a complete orthonormal set in $H$. By Lemma $1, m$ is equivalent to $\mu_{t}$ defined by $\mu_{t}(\sigma)=$ $\sum_{n=1}^{\infty}\left(1 / 2^{n}\right)\left(E(\sigma) T^{t} h_{n}, T^{t} h_{n}\right)$ for every $t$. Now 


$$
\begin{aligned}
\mu_{t}(\sigma) & =\sum_{n=1}^{\infty} \frac{1}{2^{n}}\left(E(\sigma) T^{t} h_{n}, T^{t} h_{n}\right)=\sum_{n=1}^{\infty}\left(T^{-t} E(\sigma) T^{t} h_{n}, h_{n}\right) \\
& =\sum_{n=1}^{\infty} \frac{1}{2^{n}}\left(E(\sigma+t) h_{n}, h_{n}\right)=\mu_{0}(\sigma+t) .
\end{aligned}
$$

Thus $\mu_{0}$ and $\mu_{t}$ are mutually absolutely continuous for every $t$, and so by Lemma $2 \mu_{0}$ and Lebesgue measure are mutually absolutely continuous. So $m$ and Lebesgue measure are mutually absolutely continuous.

LEMMA 5. Let $E$ be a stationary spectral measure of multiplicity 1. Then there exists a vector valued measure $z(\cdot)$ on $\mathscr{B}$ and a function $A$ on $R$ of absolute value 1 such that

(i ) $z(A) \perp z(B)$ whenever $A \cap B=$ null set,

(ii) $\|z(A)\|^{2}=L(A)$ where $L$ stands for the Lebesgue measure,

(iii) every $h \in H$ has a representation of the type

$$
h=\int_{-\infty}^{\infty} \varphi_{h}(\lambda) d z(\lambda), \int_{-\infty}^{\infty}\left|\varphi_{h}(\lambda)\right|^{2} d \lambda<\infty,
$$

(iv) $E(\sigma) h=\int_{\sigma} \varphi_{h}(\lambda) d z(\lambda)$,

(v) $A^{-1}(\lambda) A(\lambda+t)$ is measurable in $\lambda$ for every $t$,

(vi) $T^{t} h=\int_{-\infty}^{\infty} \varphi_{h}(\lambda) A^{-1}(\lambda) A(\lambda+t) d z(\lambda+t)$.

Proof. Let $w$ be a cyclic vector for $E$ and $m$ the measure defined by $m(\sigma)=\|E(\sigma) w\|^{2}, \quad \sigma \in \mathscr{B}$. Let $\rho$ denote the Radon-Nikodym derivative $m$ with respect to the Lebesgue measure $L$. Since $m$ and $L$ are mutually absolutely continuous, $\rho>0$ a.e. $(L)$.

Define $z(\cdot)$ by $z(\sigma)=\iint_{\sigma}(1 / \sqrt{\rho(\lambda)}) d w(\lambda)$. Then properties (i) and (ii) for $z(\cdot)$ are easily verified. To prove (iii), let $h \in H$. Then $h$ has a representation: $\quad h=\int_{-\infty}^{\infty} \psi_{h}(\lambda) d w(\lambda)$ with $\int_{-\infty}^{\infty}\left|\psi_{h}(\lambda)\right|^{2} d m(\lambda)<\infty$. $h=\int_{-\infty}^{\infty} \psi_{h}(\lambda) \sqrt{\rho(\lambda)}(1 / \sqrt{\rho(\lambda)}) d w(\lambda)$. Now $(1 / \sqrt{\rho(\lambda)}) d w(\lambda)$ is equal to $d z(\lambda)$. We write $\psi_{h}(\lambda) \sqrt{\rho(\lambda)}=\varphi_{h}(\lambda)$, and representation for $h$ becomes $h=\int_{-\infty}^{\infty} \varphi_{h}(\lambda) d z(\lambda)$ with $\int_{-\infty}^{\infty}\left|\varphi_{h}(\lambda)\right|^{2} d \lambda<\infty$. This proves (iii). Again $E(\sigma) h=\int_{\sigma} \psi_{h}(\lambda) d w(\lambda)=\int_{\sigma} \varphi_{h}(\lambda) d z(\lambda)$ can be seen to be true. This proves (iv). It remains to show (v) and (vi). Let us write $\widetilde{z}_{t}(\sigma)=$ $T^{t} z(\sigma-t) . \quad \widetilde{z}_{t}$ is again a countably additive measure on $\mathscr{B}$ with values in $H$. Since $E$ is stationary, $\widetilde{z}_{t}(\sigma) \in E(\sigma) H$. So $\widetilde{z}_{t}(\sigma)$ has a representation of the type: $\widetilde{z}_{t}(\sigma)=\int_{\sigma} A(t, \lambda) d z(\lambda)$ for some function $A(t, \lambda)$, measurable in $\lambda$ for every $t$. Further 


$$
\begin{aligned}
\left\|\widetilde{z}_{t}(\sigma)\right\|^{2} & =\left\|T^{t} \boldsymbol{z}(\sigma-t)\right\|^{2}=\|\boldsymbol{z}(\sigma-t)\|^{2}=L(\sigma-t) \\
& =L(\sigma)=\int_{\sigma}|A(t, \lambda)|^{2} d \lambda .
\end{aligned}
$$

Since this holds for every $\sigma,|A(t, \lambda)|=1$ for almost every $\lambda$. We can write the relation between $\widetilde{z}_{t}(\cdot)$ and $z(\cdot)$ formally as $d \widetilde{z}_{t}(\lambda+t)=$ $A(t, \lambda) d z(\lambda+t)$ which is the same as $T^{t} d z(\lambda)=A(t, \lambda) d z(\lambda+t)$. Now

$$
\begin{aligned}
T^{s+t} d z(\lambda) & =T^{s} T^{t} d z(\lambda)=T^{s} A(t, \lambda) d z(\lambda+t) \\
& =A(t, \lambda) A(s, \lambda+t) d z(\lambda+t+s) \\
& =A(s+t, \lambda) d z(\lambda+s+t) .
\end{aligned}
$$

From this equation we get $A(t, \lambda) A(s, \lambda+t)=A(s+t, \lambda)$. Thus $A(t, \lambda)$ is a cocycle. By Lemma $3 A$ is of the type $A(\lambda+t) / A(\lambda)$ for some functions $A$. We choose this $A$ for $A$ of Lemma 5. Obviously $|A(t)|=1$ for every $t$. Further $A(t, \lambda)=A^{-1}(\lambda) A(\lambda+t)$ is measurable in $\lambda$ for every $t$. This proves (v). Finally let $h=\int_{-\infty}^{\infty} \varphi_{h}(\lambda) d z(\lambda)$. Then

$$
\begin{aligned}
T^{t} h & =T^{t} \int_{-\infty}^{\infty} \varphi_{h}(\lambda) d z(\lambda)=\int_{-\infty}^{\infty} \varphi_{h}(\lambda) T^{t} d z(\lambda)=\int_{-\infty}^{\infty} \varphi_{h}(\lambda) A(t, \lambda) d z(\lambda+t) \\
& =\int_{-\infty}^{\infty} \varphi_{h}(\lambda) A^{-1}(\lambda) A(\lambda+t) d z(\lambda+t) .
\end{aligned}
$$

This proves (vi).

3. Let us return to the notation and terminology of Theorem 1 . For $f \in L_{2}(B, \mu)$, write $T^{t} f=\chi_{t} f$, where $\chi_{t}$ is a character on $B$ corresponding to the real number $t$. It is obvious that $T^{t}$ is a commutative group of unitary operators on $L_{2}(B, \mu)$. Further following two identities can be easily verified:

(A) $\quad T^{t}(H(b) \ominus H(a))=H(b+t) \ominus H(a+t)$ where $a, b(a<b)$ are any two real numbers.

(B) For any $f \in L_{2}(B, \mu),\|E(a, b] f-f\|^{2}=\left\|T^{t} E(a, b] f-T^{t} f\right\|^{2}$. (A) and (B) together imply that $E$ is a stationary spectral measure, $T^{t} E(\sigma) T^{-t}=E(\sigma+t)$.

Proof of Theorem 1. The spectral measure $E$ of Theorem 1 is stationary as shown in the above paragraph. By hypothesis $E$ has multiplicity 1. By Lemma 5 there exists a vector valued measure $z(\cdot)$ with values in $L_{2}(B, \mu)$ and a function $A$ on $R$ of absolute value 1 satisfying (i)-(vi) of Lemma 5. $\chi_{0} \in L_{2}(B, \mu)$ has a representation of the type $\chi_{0}=\int_{-\infty}^{0} f(\lambda) d z(\lambda)$.

$$
\begin{gathered}
\chi_{t}=\chi_{t} \chi_{0}=T^{t} \chi_{0}=\int_{-\infty}^{0} f(\lambda) A^{-1}(\lambda) A(\lambda+t) d z(\lambda+t) . \\
\chi_{t} A^{-1}(t)=\int_{-\infty}^{0} f(\lambda) A^{-1}(\lambda) A(\lambda+t) A^{-1}(t) d z(\lambda+t) .
\end{gathered}
$$


We shall show that $\chi_{t}(b) A^{-1}(t)$ is measurable in $t$ for almost every $b \in B$ (with respect to $\mu$ ). First observe that, by remark following Lemma $3, A^{-1}(\lambda) A(\lambda+t) A^{-1}(t)$ is measurable in $(t, \lambda)$. Next the integral representation $\left(^{*}\right)$ for $\chi_{t} A^{-1}(t)$ exists in the sense that approximating sums of the type $\sum_{i} f\left(\lambda_{i}\right) A^{-1}\left(\lambda_{i}\right) A^{-1}\left(\lambda_{i}+t\right) A^{-1}(t) z\left(\sigma_{i}\right)$ converge to $\chi_{t} A^{-1}(t)$ in $L_{2}(B, \mu)$. Since $\mu$ is a finite measure the sequence of approximating sums converges to $\chi_{t} A^{-1}(t)$ almost everywhere on $B$ with respect to $\mu$. But each of the approximating sum is measurable in $t$ for every $b \in B$. Hence for almost every $b \in B$ (with respect to $\mu$ ) $\chi_{t}(b) A^{-1}(t)$ is measurable in $t$ (see [2], p. 430). Now fix a $b^{\prime} \in B$ such that $\chi_{t}\left(b^{\prime}\right) A^{-1}(t)$ is measurable in $t$. Consider $\chi_{t}\left(b^{\prime}-b\right) . \quad \chi_{t}\left(b^{\prime}-b\right)=$ $\chi_{t}\left(b^{\prime}\right)\left(\chi_{t}(b)\right)^{-1}=\chi_{t}\left(b^{\prime}\right) A^{-1}(t)\left(\chi_{t}(b) A^{-1}(t)\right)^{-1}=$ ratio of two measurable functions in $t$ for almost every $b$. So $\chi_{t}\left(b-b^{\prime}\right)$ is measurable in $t$ for almost every $b \in B$ (with respect to $\mu$ ). But a measurable character on $R$ is necessarily continuous. So $b^{\prime}-b \in R$ for almost every $b \in B$ (with respect to $\mu$ ). Hence $b \in b^{\prime}+R$ for almost every $b \in B$ (with respect to $\mu)$. So $\mu$ sits on a coset of $R$ in $B$.

Much more is true than simply the fact that $\mu$ sits on a coset of $R$ in $B$. For example, $\mu$ restricted to the appropriate coset is absolutely continuous with respect to the Lebesgue measure on that coset and if $f$ is its Radon-Nikodym derivative, then

$$
\left|\int \frac{\log f(\lambda)}{1+\lambda^{2}} d \lambda\right|<\infty \quad \text { ([2], p. 586). }
$$

A converse of Theorem 1 is true: If $\cap H_{s}=\{0\}$ and $\mu$ sits on a coset of $R$ in $B$, then $E$ has multiplicity 1 . This is essentially a consequence of a result of 0 . Hanner [3] on representation of weakly stationary purely nondeterministic stationary stochastic process.

A finite regular measure $\nu$ on $B$ is called analytic if $\int_{B} \chi_{t}(b) \nu(d b)=0$ for $t<0$. Let $\mu$ denote the total variation measure of $\nu$. It can be shown that the subspaces $H_{s}$ in $L_{2}(B, \mu)$ have the property $\bigcap_{-\infty<s<\infty} H_{s}=$ $\{0\}$. Let $E$ be the spectral measure generated by $H_{s}-\infty<s<\infty$. If $E$ has multiplicity 1 then by Theorem $1 \mu$ sits on a coset of $R$ in $B$ and so $\nu$ sits on a coset of $R$ in $B$.

Recently in collaboration with V. Mandrekar, we have studied finite regular measure $\mu$ on $B$ for which $\bigcap_{-\infty<s<\infty} H_{s}=\{0\}$ without assuming that spectral measure $E$ has multiplicity 1 . These results will be published elsewhere.

I would like to express my sincere thanks to Professor S. Koh for explaining to me the algebraic meaning of cocycles and coboundaries, and to Professor V. Mandrekar for useful discussions. 


\section{REFERENCES}

1. H. Cramer, On the structure of purely non-deterministic stochastic processes, Arkiv for Mathematik 4 (1961), 249-266.

2. L. J. Doob, Stochastic Processes, John Wiley, New York.

3. Olaf Hanner, Deterministic and non-deterministic stationary random processes, Arkiv for Mathematik 1 (1949), 101-117.

4. Henry Helson, Lectures on Invariant Subspaces, Academic Press, New York, 1964. 5. - Compact group with ordered duals, Proc. London Math. Soc. Vol. XIVA (June, 1965), 144-156.

6. Henry Helson and David Lowdenslager, Prediction theory and Fourier series in several variables II, Acta Math. 106 (1961), 175-205.

7. —, Invariant subspaces, Proc. Int. Symp on Linear Spaces, Jerusalem, 1961, $25-62$.

8. K. de Leeuw, and I. Glicksberg, Quasi-invariance and analyticity of measures on compact groups, Acta Mathematica 109 (1963), 179-205.

9. G. W. Mackey, A theorem of Stone and Von Neumann, Duke Math. J. 16 (1949), $313-326$.

Received June 6, 1966.

WASHINGTON UNIVERSITY

ST. LOUIS, MISSOURI

UNIVERSITY OF MiNNESOTA

Minneapolis, Minnesota 


\section{PACIFIC JOURNAL OF MATHEMATICS}

\section{EDITORS}

\section{H. ROYDEN \\ Stanford University \\ Stanford, California}

\author{
J. P. JANS \\ University of Washington \\ Seattle, Washington 98105
}

J. DugundJI

Department of Mathematics

Rice University

Houston, Texas 77001

RichaRd ARENS

University of California

Los Angeles, California 90024

\section{ASSOCIATE EDITORS}
E. F. BECKENBACH
B. H. NeumanN
F. WOLF
K. YOSIDA

\section{SUPPORTING INSTITUTIONS}

\author{
UNIVERSITY OF BRITISH COLUMBIA \\ CALIFORNIA INSTITUTE OF TECHNOLOGY \\ UNIVERSITY OF CALIFORNIA \\ MONTANA STATE UNIVERSITY \\ UNIVERSITY OF NEVADA \\ NEW MEXICO STATE UNIVERSITY \\ OREGON STATE UNIVERSITY \\ UNIVERSITY OF OREGON \\ OSAKA UNIVERSITY \\ UNIVERSITY OF SOUTHERN CALIFORNIA
}

\author{
STANFORD UNIVERSITY \\ UNIVERSITY OF TOKYO \\ UNIVERSITY OF UTAH \\ WASHINGTON STATE UNIVERSITY \\ UNIVERSITY OF WASHINGTON

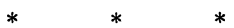 \\ AMERICAN MATHEMATICAL SOCIETY \\ CHEVRON RESEARCH CORPORATION \\ TRW SYSTEMS \\ NAVAL ORDNANCE TEST STATION
}

Mathematical papers intended for publication in the Pacific Journal of Mathematics should be typewritten (double spaced). The first paragraph or two must be capable of being used separately as a synopsis of the entire paper. It should not contain references to the bibliography. Manuscripts may be sent to any one of the four editors. All other communications to the editors should be addressed to the managing editor, Richard Arens at the University of California, Los Angeles, California 90024.

50 reprints per author of each article are furnished free of charge; additional copies may be obtained at cost in multiples of 50 .

The Pacific Journal of Mathematics is published monthly. Effective with Volume 16 the price per volume (3 numbers) is $\$ 8.00$; single issues, $\$ 3.00$. Special price for current issues to individual faculty members of supporting institutions and to individual members of the American Mathematical Society: $\$ 4.00$ per volume; single issues $\$ 1.50$. Back numbers are available.

Subscriptions, orders for back numbers, and changes of address should be sent to Pacific Journal of Mathematics, 103 Highland Boulevard, Berkeley 8, California.

Printed at Kokusai Bunken Insatsusha (International Academic Printing Co., Ltd.), 7-17, Fujimi 2-chome, Chiyoda-ku, Tokyo, Japan.

PUBLISHED BY PACIFIC JOURNAL OF MATHEMATICS, A NON-PROFIT CORPORATION

The Supporting Institutions listed above contribute to the cost of publication of this Journal, but they are not owners or publishers and have no responsibility for its content or policies. 


\section{Pacific Journal of Mathematics}

\section{Vol. 23, No. 2 \\ April, 1967}

Herbert Stanley Bear, Jr. and Bertram John Walsh, Integral kernel for

one-part function spaces .......................... 209

Mario Borelli, Some results on ampleness and divisorial schemes ....... 217

John A. Erdos, Unitary invariants for nests . ................... 229

Nathaniel Grossman, The volume of a totally-geodesic hypersurface in a pinched manifold.................................. 257

D. M. Hyman, A generalization of the Borsuk-Whitehead-Hanner theorem ............................................. 263

I. Martin (Irving) Isaacs, Finite groups with small character degrees and large prime divisors ............................. 273

I. Martin (Irving) Isaacs, Two solvability theorems ................ 281

William Lee Johnson, The characteristic function of a harmonic function in a locally Euclidean space ............................... 291

Ralph David Kopperman, Application of infinitary languages to metric spaces ............................................. 299

John Lauchlin MacDonald, Relative functor representability ............ 311

Mahendra Ganpatrao Nadkarni, A class of measures on the Bohr group.... 321

Keith Lowell Phillips, Hilbert transforms for the p-adic and p-series fields....................................... 329

Norman R. Reilly and Herman Edward Scheiblich, Congruences on regular semigroups ...

Neil William Rickert, Measures whose range is a ball ... . .

Gideon Schwarz, Variations on vector measures

Ronald Cameron Riddell, Spectral concentration for self-adjoint operators. .

Haskell Paul Rosenthal, A characterization of restrictions of

Fourier-Stieltjes transforms ................... 